\title{
Effectiveness of a 1-day aspiration plus Gamma Knife surgery procedure for metastatic brain tumor with a cystic component
}

\author{
Clinical article
}

\author{
Fumi Higuchi, M.D., ${ }^{1}$ Shunsuke Kawamoto, M.D., ${ }^{1}$ Yoshihiro Abe, M.D., ${ }^{1}$ \\ Phyo Kim, M.D., Ph.D., ${ }^{1}$ ANd Keisuke Ueki, M.D. ${ }^{1,2}$ \\ ${ }^{1}$ Department of Neurosurgery and ${ }^{2}$ Comprehensive Cancer Center, Dokkyo Medical University, Mibu, \\ Tochigi, Japan
}

Object. Gamma Knife surgery (GKS) has gained increasing relevance in the treatment of metastatic brain tumors, but many metastatic tumors contain a large cystic component and often exceed the size limit for GKS. For such lesions, the authors adopted a procedure in which stereotactic aspiration is first performed and followed immediately by GKS on the same day. In this paper, the authors describe this 1-day combined procedure and evaluate its efficacy.

Methods. Between 2005 and 2010, 25 cystic metastases in 25 patients were treated at Dokkyo Medical University. The patients first underwent MRI and stereotactic aspiration of the cyst while stationary in a Leksell stereotactic frame; immediately afterward, the patients underwent a second MR imaging session and Gamma Knife treatment. Tumor volume reduction, tumor control rate, and overall survival were examined.

Results. Tumor volume, including the cystic component, decreased from $8.0-64.2 \mathrm{~cm}^{3}$ (mean $20.3 \mathrm{~cm}^{3}$ ) to 3.0 $36.2 \mathrm{~cm}^{3}$ (mean $10.3 \mathrm{~cm}^{3}$ ) following aspiration, and the volume of 24 of 25 lesions decreased to less than $16.6 \mathrm{~cm}^{3}$, which is equivalent to the volume of a 3.16-cm sphere. At least $20 \mathrm{~Gy}$ was delivered to the entire lesion in 24 of 25 cases. Good tumor control was obtained in 16 of 21 cases that could be evaluated during a median follow-up period of 11 months (range 1-27 months); however, reaccumulation of cyst contents was observed in 2 patients who required Ommaya reservoir placement.

Conclusions. The 1-day aspiration plus GKS procedure is an effective and time-efficient treatment for large cystic brain metastases.

(http://thejns.org/doi/abs/10.3171/2012.7.GKS121001) \section{KEY WoRDS • brain metastasis $\bullet \quad$ stereotatic radiosurgery
stereotactic cyst aspiration}

$\mathrm{G}$ AMMA Knife surgery has gained increasing relevance in the treatment of metastatic brain tumors. ${ }^{3,5,6}$ It allows treatment of lesions located at deep, surgically inaccessible locations as well as treatment of several lesions at the same time if necessary. In addition, GKS is less invasive than other treatment modalities and, therefore, more feasible for patients with cancer, who usually need to continue treatment for the primary disease at an advanced stage. On the other hand, GKS has its own limitations: it is not suitable for large lesions, in which risk of an acute radiation effect is high. Lesions larger than $3 \mathrm{~cm}$ in diameter are generally con-

Abbreviations used in this paper: GKS = Gamma Knife surgery; $\mathrm{WBRT}=$ whole-brain radiation therapy. sidered poor candidates for GKS and are deemed more appropriate for resection, if possible, or for fractionated external beam radiation therapy. 5,6

Many brain metastases contain cystic components, and tumors with a large cyst are challenging lesions to manage. Complete surgical removal of the thin cyst wall containing tumor cells without damaging brain tissue is technically difficult, while the volume of the whole lesion, including the cyst wall, often exceed the size limit for GKS, even when the solid portions of tumor are small. For such lesions, we have been adopting a 2-step procedure: performing stereotactic cyst fluid aspiration, using a Leksell frame (Elekta AB), followed by GKS on the same day using the same frame. Here we describe the details of the procedure, a 1-day aspiration plus GKS protocol, 
and present the results of this treatment option, which we believe is worth considering when feasible.

\section{Methods}

Between February 2005 and April 2010, 517 patients with brain metastases underwent GKS at Dokkyo Medical University Hospital. Of those patients, 25 harbored 25 metastatic brain tumors with cystic components. These 25 patients underwent stereotactic aspiration followed by GKS, and their cases were retrospectively analyzed. The diagnosis of brain metastasis was based on findings on MRI studies. There were 13 men and 12 women, ranging in age from 32 to 77 years (mean 58 years). The distribution of primary lesions consisted of lung cancer in 11 patients, breast cancer in 7 patients, colon cancer in 3 patients, and other malignancies in 4 patients. The cystic tumors were located in the frontal lobe in 5 patients, parietal lobe in 7 patients, occipital lobe in 5 patients, temporal lobe in 1 patient, basal ganglia in 2 patients, and cerebellum in 5 patients. The Recursive Partitioning Analysis class was I in 1 patient, II in 15 patients, and III in 7 patients, indicating that approximately one-quarter of the patients had a poor prognosis (Table 1).

The treatment procedure was performed in the following manner. After a local anesthetic agent had been applied to the head, a Leksell stereotactic frame was applied using fine adjustments and taking into account the location of the lesion and the presumed path for stereotactic aspiration. Magnetic resonance images were obtained, and the patient was immediately transferred to the operating room. The target, which usually was the center of the cyst, and the path for the aspiration needle were determined using Surgiplan software. The patient was placed in an appropriate position and given a local anesthetic agent before stereotactic needle aspiration commenced. Aspiration continued until no more fluid was aspirated, and the wound was closed without placement of a reservoir or drainage tubes. The patient then underwent Gdenhanced MRI, on the basis of which dose planning was made, and then GKS. The patient was usually discharged on the following day. Effort was made to deliver a prescribed dose of $\geq 20$ Gy to the entire lesion (Figs. 1, 2, and 3).

We measured tumor volume before and after aspiration, and calculated the reduction in tumor volume. After GKS, follow-up MR images with contrast enhancement were obtained every 2 or 3 months. With respect to the treatment results, the tumor was considered to be controlled if the size of the enhancing lesion had either decreased or stabilized in size compared with the pretreatment assessment. Any treatment-associated complication was recorded.

\section{Results}

\section{Volume Reduction Due to Stereotactic Aspiration}

The initial tumor target volumes, including the cystic component, ranged from 8.0 to $64.2 \mathrm{~cm}^{3}$ (mean $20.3 \mathrm{~cm}^{3}$ ). The postaspiration tumor volumes, which constituted the target volumes for GKS, ranged from 3.0 to $36.2 \mathrm{~cm}^{3}$
TABLE 1: Clinical characteristics in 25 patients with metastatic tumors*

\begin{tabular}{|c|c|}
\hline Characteristic & No. of Patients \\
\hline \multicolumn{2}{|l|}{ age } \\
\hline$<65$ yrs & 20 \\
\hline$>65$ yrs & 5 \\
\hline \multicolumn{2}{|l|}{ sex } \\
\hline male & 13 \\
\hline female & 12 \\
\hline \multicolumn{2}{|l|}{ primary tumor } \\
\hline lung & 11 \\
\hline breast & 7 \\
\hline colon & 3 \\
\hline kidney & 1 \\
\hline liver & 1 \\
\hline ovary & 1 \\
\hline unknown & 1 \\
\hline \multicolumn{2}{|l|}{ location of lesions } \\
\hline frontal lobe & 5 \\
\hline occipital lobe & 5 \\
\hline parietal lobe & 7 \\
\hline basal ganglia & 2 \\
\hline temporal lobe & 1 \\
\hline posterior fossa & 5 \\
\hline \multicolumn{2}{|l|}{ RPA classification } \\
\hline Class I & 1 \\
\hline Class II & 15 \\
\hline Class III & 7 \\
\hline unknown & 2 \\
\hline
\end{tabular}

* $\mathrm{RPA}=$ recursive partitioning analysis.

(mean $10.3 \mathrm{~cm}^{3}$ ). These numbers demonstrate significant volume reduction-roughly 50\% -in most cases (Fig. 4). Before aspiration, 12 lesions exceeded $14 \mathrm{~cm}^{3}$ in volume, which is equivalent to a 3 -cm-diameter sphere. In 6 of those 12 lesions, the target volume was reduced to less than $14 \mathrm{~cm}^{3}$ due to aspiration. In 24 of 25 cases, the target volumes were reduced to $\leq 16.6 \mathrm{~cm}^{3}$, which was equivalent to the volume of a $3.16-\mathrm{cm}$-diameter sphere, after aspiration (Table 2).

\section{Tumor Control by GKS}

A prescribed dose of $\geq 20$ Gy was delivered to 24 (96\%) of the 25 lesions. Follow-up periods in the study population ranged from 1 to 27 months (median 11 months). Four patients were excluded from the analysis because they died of nonneurological causes before their first follow-up MRI examination. In 16 (76\%) of the remaining 21 patients, the tumors were controlled well at the end of follow-up or at the time of the patient's death from nonneurological causes. In those patients, 5 tumors recurred between 7 and 17 months (mean 9 months) after treatment. Of those lesions, 3 tumors received salvage therapy: 2 were treated with repeated GKS and 1 tumor 

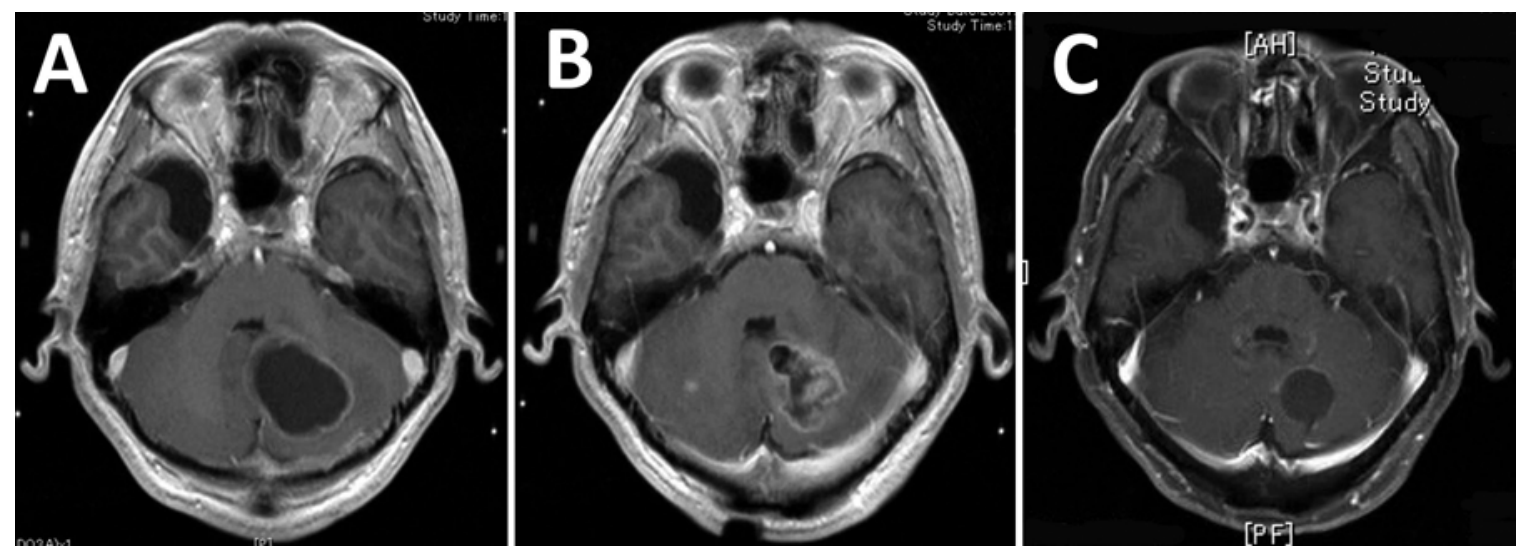

FIG. 1. Case 14. Effect of aspiration. A: Preaspiration Gd-enhanced T1-weighted MR image showing a cystic round mass with a thin cyst wall located in the left cerebellum. B: Postaspiration Gd-enhanced T1-weighted MR image demonstrating marked shrinkage of the cyst, leading to a significant reduction in the GKS targeted volume (from 34 to $10.2 \mathrm{~cm}^{3}$ ). C: Follow-up MR image obtained 10 months after GKS showing good control of the lesion.

was resected. Two other patients were offered supportive care for poor conditions. In those patients, 3 tumors received salvage therapy: 2 were treated with additional GKS, and 1 tumor was resected. In two patients, the cystic components of the lesion increased in size and required placement of an Ommaya reservoir soon after treatment: 2 weeks post-GKS in 1 patient and 3 weeks post-GKS in the other. Cyst aspiration was necessary only once in the former patient, and a second aspiration was required 1 month after initial placement of the Ommaya reservoir in the latter. No further aspiration was necessary. Because the tumor mass itself was controlled, these cases were not considered to have had treatment failure. There was no significant complication related to the stereotactic aspiration procedure, except for intraoperative seizure events observed in 2 patients. One patient whose primary tumor was a renal cell carcinoma developed intratumoral hemorrhage 9 months after the procedure.

For all patients, the 1- and 2-year Kaplan-Meier estimated survival rate was $43.1 \%$ and $14.4 \%$, respectively (Fig. 5). During follow-up, 19 patients died, 1 patient remained alive, and 5 patients were lost to follow-up. Of the 19 patients who died, only 3 patients displayed significant neurological progression.

\section{Discussion}

Gamma Knife surgery has been established as a powerful tool in the management of metastatic brain tumors. When feasible, it delivers good tumor control equivalent to that of open resection and WBRT, but with much less invasiveness; this minimal invasiveness is an important advantage in the care of patients with advanced systemic cancer, who occasionally have to undergo further treatment during the course of their disease. $3,6,9$ The indication for radiosurgery, however, is limited by several factors, most frequently by the size and location of the tumor. With respect to size limits, a tumor exceeding $3 \mathrm{~cm}$ in diameter is generally considered unsuitable for GKS because the risk of an acute brain reaction to high-dose radiation is unacceptably high. On the other hand, many large metastatic lesions contain a large cystic portion that does not contain tumor cells, despite constituting most of the lesions' mass. Such lesions pose an challenge for neurosurgeons for 3 reasons: 1 ) resection of the thin layer containing tumor cells is not easy; 2) the size of the tumor easily exceeds the limit for GKS; and 3) WBRT often does not provide immediate mass reduction and resolution of neurological symptoms. When large cystic lesions are located deep in the brain or in eloquent areas, WBRT generally may be the treatment of choice. Some papers
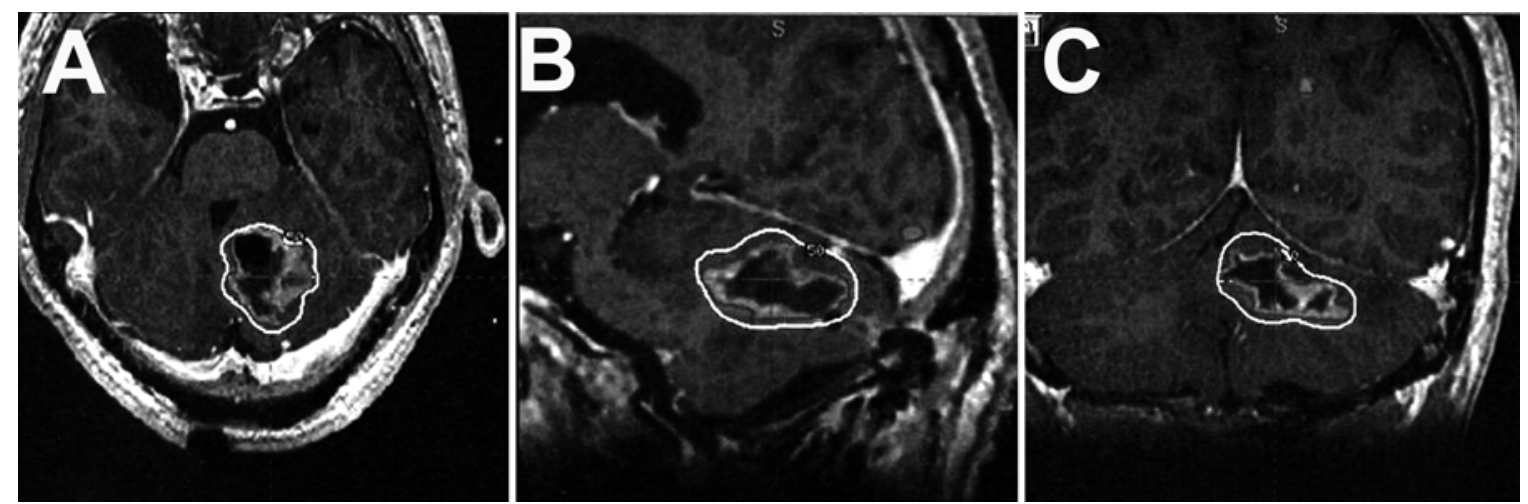

FIG. 2. Case 14. Actual dose planning using axial (A), sagittal (B), and coronal (C) MR images. The white line represents the $50 \%, 23-G y$ prescribed dose line. 

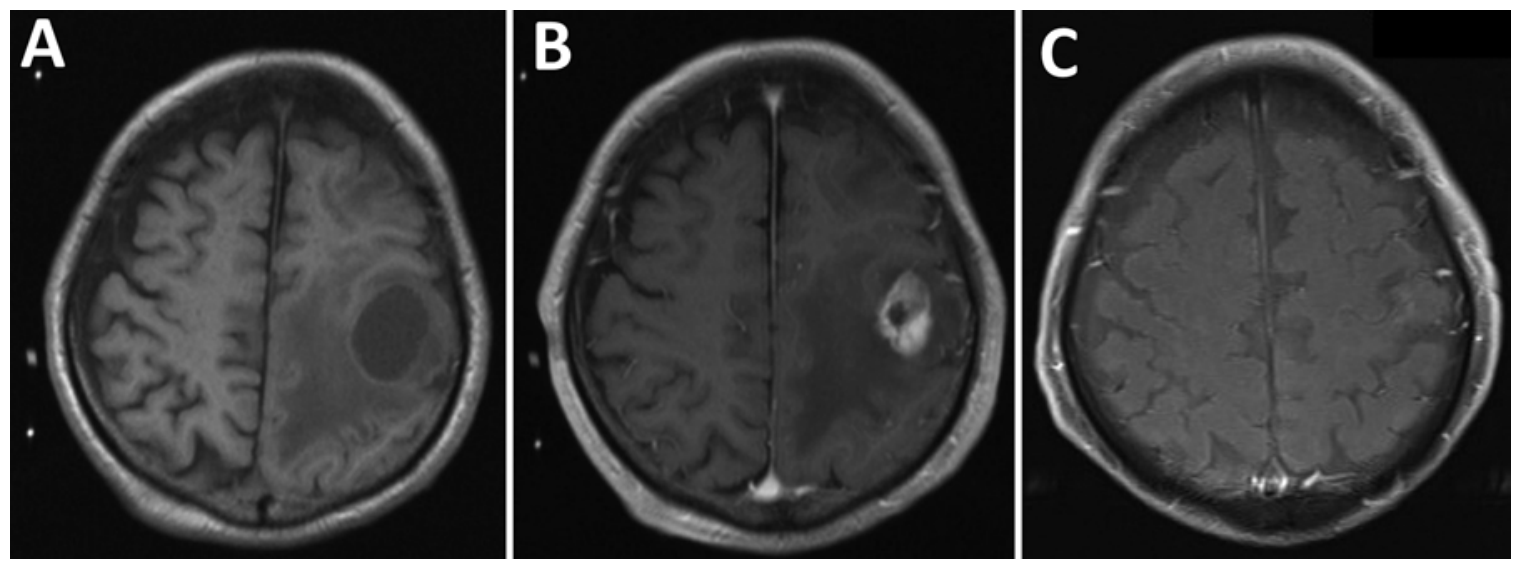

FIG. 3. Case 5. An example of successful treatment of a metastatic tumor in the brain using the 1-day aspiration plus GKS protocol. A: Preaspiration MR image showing a large cystic mass measuring $17.1 \mathrm{~cm}^{3}$ in the left motor cortex. B: Shrinkage of the cyst to $7.2 \mathrm{~cm}^{3}$ after aspiration. C: Follow-up MR image obtained 15 months after GKS showing a complete response.

have reported that such lesions are difficult to manage and are associated with an insufficient control rate.,9,

In search of the best treatment options for such large cystic metastatic tumor, we designed a sequence of procedures in which stereotactic cyst aspiration is immedi- ately followed by GKS. By doing so, the target volume for GKS is reduced, enabling delivery of a sufficient prescribed dose to the tumor cells, which are usually located at the cyst wall and mural nodules. Use of the same Leksell frame for both stereotactic aspiration and GKS al-

TABLE 2: Results of 1-day aspiration plus GKS in 25 patients with metastatic tumors*

\begin{tabular}{|c|c|c|c|c|c|c|c|c|c|c|}
\hline \multirow[b]{2}{*}{ Case } & \multirow[b]{2}{*}{ Age (yrs), Sex } & \multirow[b]{2}{*}{ Primary Cancer } & \multirow[b]{2}{*}{ Location of Met } & \multicolumn{2}{|c|}{ Target Vol $\left(\mathrm{cm}^{3}\right)$} & \multirow[b]{2}{*}{$\operatorname{VR}\left(\mathrm{cm}^{3}\right)$} & \multirow[b]{2}{*}{$\mathrm{AFV}(\mathrm{ml})$} & \multirow{2}{*}{$\begin{array}{c}\text { Margin } \\
\text { Dose (Gy) }\end{array}$} & \multirow[b]{2}{*}{ PFS (mos) } & \multirow[b]{2}{*}{ OS (mos) } \\
\hline & & & & Pre-Asp & Post-Asp & & & & & \\
\hline 1 & $65, M$ & breast & parietal & 8 & 7.8 & 0.2 & 6 & 23 & 10 & 10 \\
\hline 3 & $63, M$ & kidney & occipital & 64.2 & 15.3 & 48.9 & 60 & 22 & 11 & 11 \\
\hline 4 & $47, \mathrm{~F}$ & lung & basal ganglia & 9.8 & 7 & 2.8 & 7 & 23 & 12 & 12 \\
\hline 7 & $62, \mathrm{M}$ & lung & frontal & 30.9 & 15.9 & 15 & 20 & 23 & 9 & 17 \\
\hline 8 & $75, \mathrm{M}$ & stomach & frontal & 11.6 & 4.5 & 7.1 & 12 & 23 & 1 & 1 \\
\hline 9 & $76, \mathrm{M}$ & lung & cerebellum & 12.5 & 5.9 & 6.6 & 5 & 23 & 1 & 1 \\
\hline 10 & $74, \mathrm{M}$ & unknown & frontal & 13.4 & 6.9 & 0.5 & 15 & 22 & 1 & 1 \\
\hline 11 & $69, \mathrm{~F}$ & lung & cerebellum & 11.2 & 10.4 & 0.8 & 10 & 23 & 1 & 1 \\
\hline 15 & $63, M$ & lung & frontal & NA & 5.3 & NA & 11 & 20 & 7 & 23 \\
\hline 16 & $45, \mathrm{~F}$ & breast & cerebellum & 8.8 & 3 & 5.8 & 8 & 10 & 8 & 24 \\
\hline 17 & $63, \mathrm{~F}$ & breast & parietal & 12 & 6.6 & 5.4 & NA & 22 & 17 & 27 \\
\hline 18 & $43, \mathrm{~F}$ & breast & occipital & 31.1 & 8.8 & 22.3 & 10 & 23 & 27 & 27 \\
\hline 19 & $44, \mathrm{M}$ & liver & basal ganglia & 14.9 & 12.8 & 1.2 & 5 & 20 & 3 & 3 \\
\hline 20 & $54, \mathrm{M}$ & colon & occipital & 42.9 & 36.2 & 6.7 & 50 & 20 & 1 & 4 \\
\hline 21 & $32, F$ & breast & temporal & 10.9 & 6.3 & 4.6 & 10 & 22 & 4 & 4 \\
\hline 22 & $51, \mathrm{~F}$ & ovary & parietal & 12.1 & 7.5 & 4.6 & 5 & 23 & 5 & 5 \\
\hline 23 & $68, F$ & colon & cerebellum & 20.7 & 10.8 & 9.9 & 10 & 23 & 5 & 5 \\
\hline 24 & $64, \mathrm{~F}$ & breast & occipital & 28 & 15.7 & 12.3 & 20 & 20 & 5 & 5 \\
\hline
\end{tabular}

* AFV = aspirated fluid volume; Met = metastasis; NA = not available; OS = overall survival; PFS = progression-free survival; Post-Asp = postaspiration; Pre-Asp = preaspiration; VR = volume reduction achieved by aspiration. 


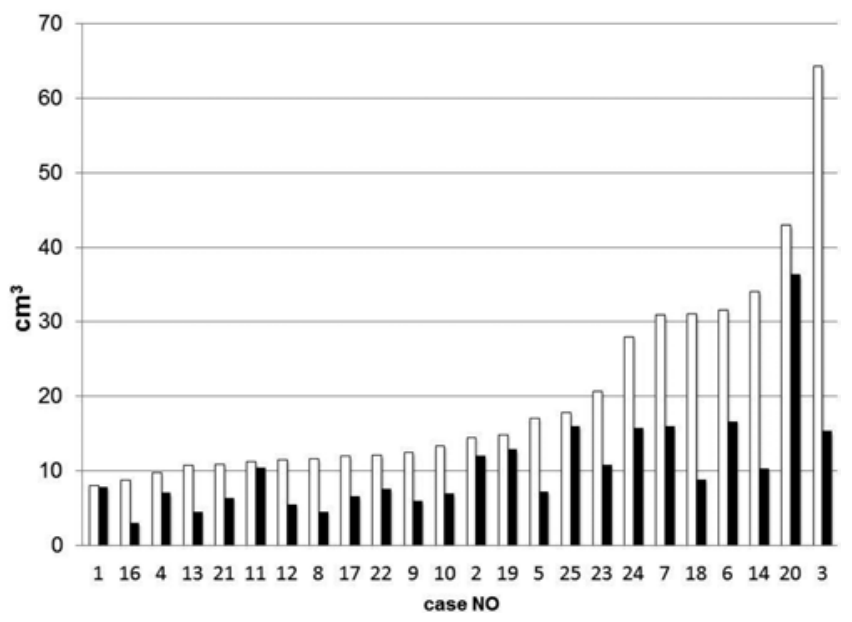

FIG. 4. Bar graph showing the effect of aspiration in each case. Open bars indicate preaspiration volumes and black bars indicate postaspiration volumes.

lows a seamless flow of procedures performed in 1 day. In most cases, we could achieve a good volume reduction, facilitating a sufficient treatment dose delivery to tumorcontaining tissue. It is fairly well established that in GKS for brain metastasis, tumor volumes inversely correlate with local tumor control rates and patient survival. Many studies have reported high tumor control rates around $90 \%,{ }^{8}$ but the local tumor control rate and patient survival decline as tumor volumes increase, especially when the lesions exceed $3 \mathrm{~cm}$ in diameter., ${ }^{1,911,15}$ The reason for poor control of large tumors by GKS is an inadequate prescribed dose. Shehata et al. ${ }^{13}$ reported a significant decline in the tumor control rate in cases treated with $<20$ Gy, whereas they found no such difference in the results of patients treated by prescribed doses $>20$ Gy. Based on such data, those authors proposed $20 \mathrm{~Gy}$ as the minimal prescribed dose for the maximal effect. On the other hand, a high prescribed dose and a large target volume are associated with an increased risk of radiation necrosis. ${ }^{4,7}$ The Radiation Therapy Oncology Group proposed that the maximum tolerated doses of single-fraction radiosurgery should be as follows: 24 Gy for tumors $<20 \mathrm{~mm} ; 18$ Gy for tumors $21-30 \mathrm{~mm}$; and 15 Gy for tumors $31-40$ $\mathrm{mm}$ in maximum diameter. ${ }^{12}$ By reducing the target volume by performing cyst aspiration immediately before GKS, we could deliver a prescribed dose of $\geq 20$ Gy in all but 1 case, in which a lower dose was prescribed because

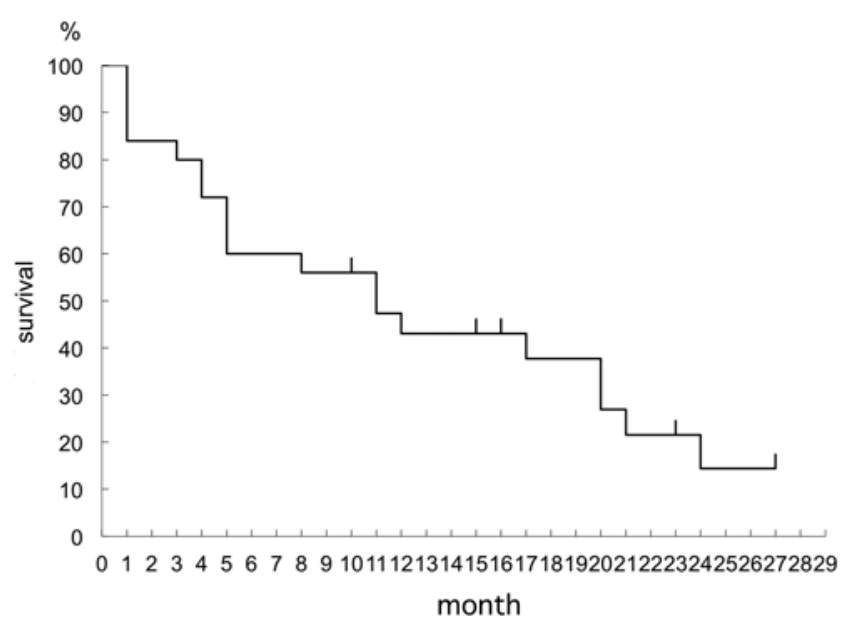

Fig. 5. Kaplan-Meier survival curve for all 25 patients.

the patient had previously received radiation treatment for cerebellar lesions. In summary, the aspiration procedure effectively reduced target volumes, resolved the mass effect, and enabled an adequate prescribed dose during GKS, leading to good tumor control without acute radiation effects.

The stereotactic aspiration procedure was safe, and we did not experience any significant complications, such as hemorrhage, infection, and along-the-tract dissemination, even in relatively deep-seated lesions. Although two patients experienced intraoperative seizures, they did not develop new neurological deficits.

There are a few studies describing cyst aspiration for brain metastasis prior to radiosurgery; in these, the methods and timing of the aspiration are varied (Table 3). ${ }^{2,10,14,16}$ In most studies, a drainage tube or Ommaya reservoir was placed during cyst aspiration and radiosurgery proceeded later. However, the volume reduction rate and tumor control rates in such studies did not differ significantly from ours. Yamanaka et al. ${ }^{16}$ placed a drainage tube or Ommaya reservoir during cyst aspiration and followed this procedure by radiosurgery up to 60 days later. Such a two-step approach is less intensive and may be more feasible at most institutions, although it may cost more time to patients and also may increase the risk of cyst fluid reaccumulation during the longer waiting period. The 1-day approach described in our series allows both stereotactic aspiration and GKS to be performed with a single frame application, reducing the physical and psychological burden for the patient. Use of a Leksell frame provides an ac-

TABLE 3: Summary of other series describing cyst aspiration for brain metastasis prior to radiosurgery*

\begin{tabular}{|c|c|c|c|c|c|c|c|}
\hline Authors \& Year & $\begin{array}{c}\text { No. of } \\
\text { Patients }\end{array}$ & $\begin{array}{l}\text { Mean Pre-Asp Vol } \\
\text { in } \mathrm{cm}^{3} \text { (range) }\end{array}$ & Vol Reduc (\%) & $\begin{array}{l}\text { Mean Prescribed } \\
\text { Dose in Gy (range) }\end{array}$ & 1-Day Procedure & Device† & $\begin{array}{l}\text { Local Tumor } \\
\text { Control (\%) }\end{array}$ \\
\hline Yamanaka et al., 2006 & 22 & $33.5(10.3-102.1)$ & NA & $16(8-20)$ & no & yes & 67.9 \\
\hline Park et al., 2009 & 24 & $23.2(7.9-100.9)$ & 22.1 & $20.2(13-25)$ & yes & yes & 79.2 \\
\hline present study & 25 & $20.0(8.0-64.2)$ & 51.5 & $23(13-23)$ & yes & no & 76 \\
\hline
\end{tabular}

* $N A=$ not available; Vol Reduc = volume reduction.

$\dagger$ Device = Ommaya reservoir or drainage tube placed during procedure . 
curate stereotactic procedure that enables safer aspiration of deep-seated, relatively small cystic lesions. Another advantage would be to minimize the time window within which the cyst can reaccumulate fluid and negate the need for continuous aspiration through an Ommaya reservoir. It is still possible that the cyst shape may change during GKS, but the good control rate in our series seems to suggest that coverage of the tumor volume was sufficient.

In two cases, there was rapid reaccumulation of cyst fluid after treatment, requiring placement of an Ommaya reservoir. Interestingly, the size did not change any further after Ommaya reservoir placement, and the patients did not need any additional treatment. As for tumor volume reduction, insertion of an Ommaya reservoir or a drainage tube prior to GKS was not necessary, although it was required to control cyst fluid reaccumulation after treatment in some patients. At this point, we do not have measures to predict the risk of reaccumulation in each case, but the frequency seems to be sufficiently low to adopt the 1-day aspiration plus GKS protocol if indicated.

\section{Conclusions}

One-day aspiration plus GKS is effective and less invasive than resection or WBRT for the treatment of brain metastases with a large cystic component. This strategy is the least invasive procedure for patients. Stereotactic aspiration reduces tumor volume significantly and transforms large cystic tumors into lesions more suitable for GKS.

\section{Disclosure}

The authors report no conflict of interest concerning the materials or methods used in this study or the findings specified in this paper.

Author contributions to the study and manuscript preparation include the following. Conception and design: Ueki, Higuchi, Kawamoto, Kim. Acquisition of data: Higuchi, Kawamoto, Abe. Analysis and interpretation of data: Ueki, Higuchi. Drafting the article: Higuchi. Critically revising the article: all authors. Reviewed submitted version of manuscript: all authors. Approved the final version of the manuscript on behalf of all authors: Ueki. Administrative/ technical/material support: Kawamoto, Kim. Study supervision: Kim.

\section{References}

1. Alexander E III, Moriarty TM, Davis RB, Wen PY, Fine HA, Black PM, et al: Stereotactic radiosurgery for the definitive, noninvasive treatment of brain metastases. J Natl Cancer Inst 87:34-40, 1995

2. Franzin A, Vimercati A, Picozzi P, Serra C, Snider S, Gioia L, et al: Stereotactic drainage and Gamma Knife radiosurgery of cystic brain metastasis. J Neurosurg 109:259-267, 2008

3. Gerosa M, Nicolato A, Foroni R, Zanotti B, Tomazzoli L, Miscusi $\mathrm{M}$, et al: Gamma knife radiosurgery for brain metastases: a primary therapeutic option. J Neurosurg 97 (5 Suppl): 515-524, 2002

4. Han JH, Kim DG, Chung HT, Paek SH, Park CK, Jung HW:
Radiosurgery for large brain metastases. Int J Radiat Oncol Biol Phys 83:113-120, 2012

5. Kalkanis SN, Kondziolka D, Gaspar LE, Burri SH, Asher AL, Cobbs CS, et al: The role of surgical resection in the management of newly diagnosed brain metastases: a systematic review and evidence-based clinical practice guideline. J Neurooncol 96:33-43, 2010

6. Linskey ME, Andrews DW, Asher AL, Burri SH, Kondziolka D, Robinson PD, et al: The role of stereotactic radiosurgery in the management of patients with newly diagnosed brain metastases: a systematic review and evidence-based clinical practice guideline. J Neurooncol 96:45-68, 2010

7. Majhail NS, Chander S, Mehta VS, Julka PK, Ganesh T, Rath GK: Factors influencing early complications following Gamma Knife radiosurgery. A prospective study. Stereotact Funct Neurosurg 76:36-46, 2001

8. Maldaun MV, Aguiar PH, Lang F, Suki D, Wildrick D, Sawaya $\mathrm{R}$ : Radiosurgery in the treatment of brain metastases: critical review regarding complications. Neurosurg Rev 31:1-9, 2008

9. Pan HC, Sheehan J, Stroila M, Steiner M, Steiner L: Gamma knife surgery for brain metastases from lung cancer. J Neurosurg 102 Suppl:128-133, 2005

10. Park WH, Jang IS, Kim CJ, Kwon H: Gamma knife radiosurgery after stereotactic aspiration for large cystic brain metastases. J Korean Neurosurg Soc 46:360-364, 2009

11. Petrovich Z, Yu C, Giannotta SL, O’Day S, Apuzzo ML: Survival and pattern of failure in brain metastasis treated with stereotactic gamma knife radiosurgery. J Neurosurg 97 (5 Suppl): 499-506, 2002

12. Shaw E, Scott C, Souhami L, Dinapoli R, Kline R, Loeffler J, et al: Single dose radiosurgical treatment of recurrent previously irradiated primary brain tumors and brain metastases: final report of RTOG protocol 90-05. Int J Radiat Oncol Biol Phys 47:291-298, 2000

13. Shehata MK, Young B, Reid B, Patchell RA, St Clair W, Sims $\mathrm{J}$, et al: Stereotatic radiosurgery of 468 brain metastases $\leq 2$ $\mathrm{cm}$ : implications for SRS dose and whole brain radiation therapy. Int J Radiat Oncol Biol Phys 59:87-93, 2004

14. Uchino M, Nagao T, Seiki Y, Shibata I, Terao H, Kaneko I: [Radiosurgery for cystic metastatic brain tumor.] No Shinkei Geka 28:417-421, 2000 (Jpn)

15. Varlotto JM, Flickinger JC, Niranjan A, Bhatnagar AK, Kondziolka D, Lunsford LD: Analysis of tumor control and toxicity in patients who have survived at least one year after radiosurgery for brain metastases. Int J Radiat Oncol Biol Phys 57:452-464, 2003

16. Yamanaka Y, Shuto T, Kato Y, Okada T, Inomori S, Fujino H, et al: Ommaya reservoir placement followed by Gamma Knife surgery for large cystic metastatic brain tumors. J Neurosurg 105 Suppl:79-81, 2006

Manuscript submitted May 15, 2012

Accepted July 18, 2012.

This work was presented in abstract form at the 16th International Meeting of the Leksell Gamma Knife Society, March 25-29, 2012, in Sydney, Australia.

Please include this information when citing this paper: DOI: 10.3171/2012.7.GKS121001.

Address correspondence to: Keisuke Ueki, M.D., Department of Neurosurgery, Dokkyo Medical University, Mibu, Tochigi, 3210293, Japan. email: kueki-tky@umin.ac.jp. 